\title{
eCushion: A Textile Pressure Sensor Array Design and Calibration for Sitting Posture Analysis
}

\author{
Wenyao Xu, Member, IEEE, Ming-Chun Huang, Student Member, IEEE, Navid Amini, Member, IEEE, \\ Lei He, Senior Member, IEEE, and Majid Sarrafzadeh, Fellow, IEEE
}

\begin{abstract}
Sitting posture analysis is widely applied in many daily applications in biomedical, education, and health care domains. It is interesting to monitor sitting postures in an economic and comfortable manner. Accordingly, we present a textile-based sensing system, called Smart Cushion, which analyzes the sitting posture of human being accurately and noninvasively. First, we introduce the electrical textile sensor and its electrical characteristics, such as offset, scaling, crosstalk, and rotation. Second, we present the design and implementation of the Smart Cushion system. Several effective techniques have been proposed to improve the recognition rate of sitting postures, including sensor calibration, data representation, and dynamic time warping-based classification. Last, our experimental results show that the recognition rate of our Smart Cushion system is in excess of $85.9 \%$.
\end{abstract}

Index Terms-Textile, pressure sensor, sitting posture, calibration, cushion, non-invasive.

\section{INTRODUCTION}

\section{A. Background and Related Work}

$\mathbf{S}$ ITTING is one of the most common postures of human beings in life. It is reported that people sit for six hours each day. Sitting posture is related to many health issues, such as back pain [1], sciatica [2] and cervical spondylosis [3]; therefore, sitting posture analysis receives increasing attention in the medical community due to its extensive applications and significant impacts, such as chronic diseases [4], health education (correct sitting posture for back ache prevention [1]), and human computer interface for rehabilitation (real-time sitting posture analysis for gaming [5]).

A traditional method to analyze sitting posture is to let a patient sit on a hospital chair, and a therapist or a nurse observes the patient's sitting posture and ask questions about his feeling. In general, the normal diagnostic process will take half to one hour. However, it is still too short to obtain enough information for accurate diagnosis. Furthermore, the

Manuscript received December 15, 2012; revised March 29, 2013; accepted April 18, 2013. Date of publication April 23, 2013; date of current version August 30, 2013. The associate editor coordinating the review of this paper and approving it for publication was Prof. Zeynep Celik-Butler.

W. $\mathrm{Xu}$ is with the Computer Science and Engineering Department, SUNY Buffalo, NY 14214 USA (e-mail: wenyaoxu@buffalo.edu).

L. He and M. Sarrafzadeh are with the Electrical Engineering Department, University of California, Los Angeles, CA 90095 USA (e-mail: lhe@ee.ucla.edu; majid@cs.ucla.edu).

M.-C. Huang and N. Amini are with the Computer Science Department, University of California, Los Angeles, CA 90095 USA (e-mail: mingchuh@cs.ucla.edu; amini@cs.ucla.edu).

Color versions of one or more of the figures in this paper are available online at http://ieeexplore.ieee.org.

Digital Object Identifier 10.1109/JSEN.2013.2259589 diagnostic result has low reliability and can be biased by subjective factors from doctors to patients.

For the sake of reliable diagnosis, doctors are eager to obtain more comprehensive information from patients. Therefore, it is necessary to monitor patients $24 / 7$ and offer the most comprehensive information for doctors. Camera is commonly used sensor for sitting posture analysis. Marschall et al. [6] deployed several cameras in an office setting to record sitting postures of subjects. However, this method always makes the subjects uncomfortable as cameras record other unnecessary information of the subjects, which will be related to personal privacy. Kazuhiro et al. [7] equipped chairs with four pressure sensors for sitting posture monitoring. Compared to cameras, pressure sensor has less privacy concerns and is dedicated to record the sitting pressure distribution only. However, a handful of pressure sensors are not enough to obtain the comprehensive sitting information, and on the other hand deploying more pressure sensors will increase the related cost. Currently, the community is still seeking a low-cost, highaccuracy, and reliable solution to analyze sitting postures.

\section{B. Textile Sensor}

Electrical textile, shorthand as eTextile, is a composite yarn made of fibers coated with conductive polymer. Its natural structure is loose and its inside fibers are air gapped. Considering its promising electrical characteristics, eTextile has become widely-used in fabricating sensors for diverse applications, such as pressure [8]-[10], stretch [11]-[13], strain [14], humanity sensors [15], and respiratory sensors [16]-[18]. Moreover, it can be used to measure human physiological signals for health care applications, such as EKG [19], blood pressure [20], and rehabilitation [21]. Different from silicon or piezoelectric based sensors [22], [23], eTextile offers much more comfortable touch feeling and economical price. Therefore, it can be unobtrusively integrated into apparels for daily use. For example, Mattmann et al. [24] presented a garment prototype using textile strain sensors to recognize upper body positions. Mitchell et al. [25] introduced an interactive system using a wearable textile sensor to monitor breathing patterns of human beings. Shu et al. [26] built textile pressure sensor arrays in a shoe for gait analysis.

However, the characteristics of a textile sensor are not well-described. Normally, a textile sensor suffers from several instable factors such as environmental noise, offset, scaling, and crosstalk. Especially, whenever it is integrated into clothes 


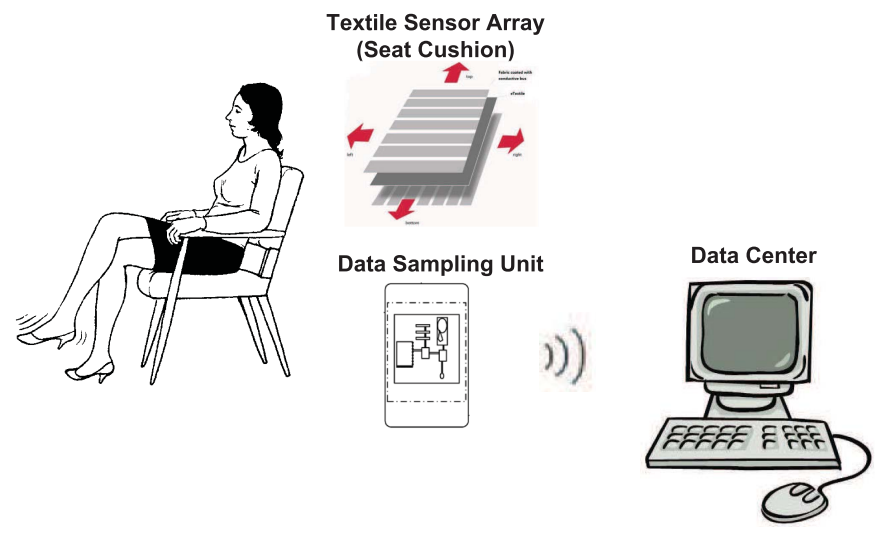

Fig. 1. The Smart Cushion architecture.

or facilities, other uncertainties from the environment will make the signal too fuzzy to be understood. For instance, in sitting monitoring application, pressure distribution is not only related to the subject's sitting posture, but also depends on other factors such as body size, weight, and sitting orientation. Therefore, signal processing and analysis on textile sensors is a challenging research topic.

\section{Contribution}

In this paper, we introduce a smart wearable system, called Smart Cushion, to analyze the sitting posture of users in daily life. Different from the traditional medical devices, Smart Cushion is based on textile material and is capable of recording patients information non-invasively. We model the textile sensor array and propose an effective framework to tackle the challenges in sitting posture analysis. The contributions of this paper are three-folds. First, we develop a textile sensor based cushion system to sense human sitting postures. The system consists of textile sensor array, data aggregator, and data analysis module. Secondly, we propose a new model to characterize and calibrate the textile sensor array. This model addresses the major issues of textile sensors, such as background noise, offset, scaling, and cross talking. Second, we present an efficient algorithm for sitting posture recognition. The algorithm reduces the dimensionality of sensor data and analyzes sitting postures based on dynamic time warping. We evaluate the performance of Smart Cushion system with 14 subjects. The experimental results show that the recognition rate can reach up to $85.9 \%$.

The remainder of this paper is organized as follows. Section II presents the overall structure and the key components of Smart Cushion system. Section III elaborates on the design of the textile sensor array. Also, we introduce the significant uncertain factors in sensor signal processing. Section IV describes the new proposed sensor calibration method. This method is general for textile sensor calibration and not restricted to any specific application. Section V discusses the algorithm of sitting posture analysis. Experimental results are shown in Section VI, and the paper is concluded in Section VII.

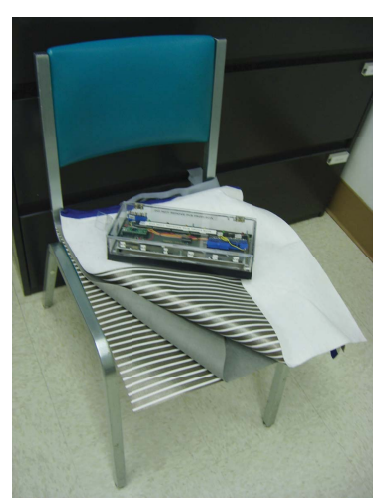

(a)

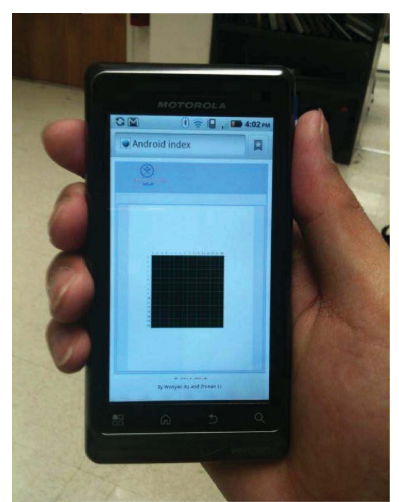

(b)
Fig. 2. The prototype of Smart Cushion. (a) Textile sensor array. (b) Smart phone.

\section{SYSTEM DESCRIPTION}

Smart Cushion is developed to be used in hospital settings and daily use, such as at home or in the office. Therefore, the essential problem is how to make the system convenient to access, straightforward to deploy, and unobtrusive to use. In addition, the system is low-cost and compatible with existing computer systems found in hospitals or homes. Fig. 1 shows the architecture of the Smart Cushion system. Basically the system is composed of three components: textile sensor array, data sampling unit and a data center. Textile sensor array captures sitting pressure distribution of the user when he sits on Smart Cushion, data sampling unit acquires the sensor values and it wirelessly transmits the data to a data center. The data center stores and analyzes the sensed data for health status monitoring.

Fig. 2(a) shows the implementation of the textile sensor array in Smart Cushion. The total sensor surface area is 10 by 10 inch, where the area of each square sensor is $5 / 8$ by $5 / 8$ inch as each row and column bus is $5 / 8$ inch wide, and the space between sensors is $1 / 8 \mathrm{inch}$. The design structure of the sensor array will be introduced in detail in Section III. The data sampling unit is designed based on Arduino Dev. board. A bluetooth module is built on the board to enable wireless data transmissions. A driver circuit is around the Arduino microcontroller for sensor array scanning. The sampling frequency is adjustable. Given that the frequency of sitting posture changes is lower than other body movements, the default data rate in Smart Cushion is set to $10 \mathrm{~Hz}$. Data storage and analysis are performed in the date center, including sitting posture analysis, human movement indexing, and statistics of motion. Considering the accessibility for mobile users, as shown in Fig. 2(a) a user-friendly application is also developed for smart phones, which conveniently displays realtime feedback of the sitting pressure distribution and facilitates continuous visualization of sitting postures.

\section{Textile Sensor ArRay Design}

eTextile is a fiber-based yarn which is coated with piezoelectric polymer [27]. The initial resistance between the top-bottom surfaces is high. When extra force is applied on the surface of the eTextile, the intra fibers will be squeezed 


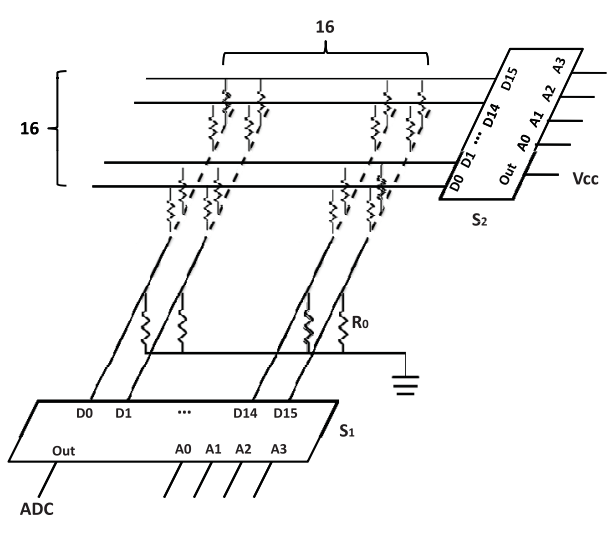

Fig. 3. The peripheral circuit for pressure distribution scanning.

together and the throughout resistance become decrease. Therefore, we can take advantage of this electrical characteristics and design a high-density and low-cost pressure sensor array.

\section{A. Sensor Array Architecture}

One type of the textile sensor sheet has been implemented in [27]. In that structure, each sensor needs an independent analog to digital channel (ADC) to sample the pressure. For example, to build an $N$ by $N$ sensor array, $N \times N I / O$ pins are required. This is prohibitive for large-scale sensing applications. In Smart Cushion, we use a three-stacked-layer structure to build the sensors, where the middle layer is eTextile. The advantage of this structure is that the top layer and the bottom layer are normal fabric uniformly coated with parallel conductive buses. As shown in Fig. 3, the conductive buses on the top layer are orthogonal to those on the bottom. The intersection area in between of the orthogonal buses is the sensing unit. Fig. 4(a) shows the sensor implementation. The top layer and bottom layer are both coated with 16 buses. Therefore, the number of sensors located on this sheet is 256. Moreover, with regards to Fig. 4(b) the total thickness is $1.5 \mathrm{~mm}$ only, which makes Smart Cushion flexible and thin enough for noninvasive use. This $N \times N$ sensor structure only requires $2 N I / O$ pins. The functionality of this circuit will be discussed in the following subsection.

\section{B. Scanning Method}

The peripheral circuit shown in Fig. 3 is used to scan the zebra pattern sensor array. Each conductive bus on the bottom is connected to an ADC from analog switch module $S_{1}$ to ground via an offset resistor $R_{0}$. Each conductive bus on the top is connected to a voltage supply via analog switch model $S_{2}$. Both $S_{1}$ and $S_{2}$ are used together in to determine which sensor is selected, and the scanning sequence is synchronized by a microcontroller. For example, when $S_{2}$ connects bus $i$ on the top layer to a voltage supply and $S_{1}$ connects bus $j$ to ADC, Smart Cushion will read the sensor located in row $i$, column $j$, which is denoted as $V_{i j}$. Therefore, this peripheral circuit has random accessability for an arbitrary sensor in the system.

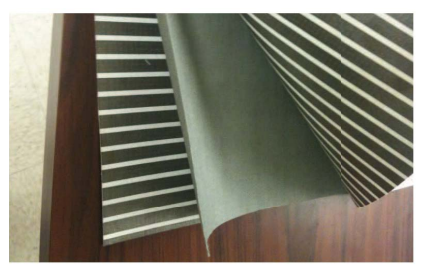

(a)

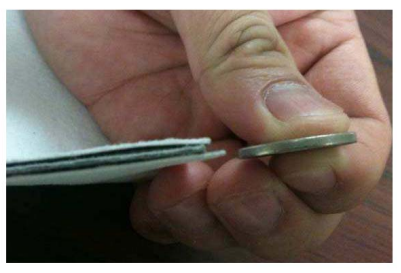

(b)
Fig. 4. The structure of textile sensor and sensor array design. (a) Sensor implementation. (b) Sensor thickness.

\section{Uncertainties in Textile Sensor Data}

When a user sits and applies forces on the sensor sheet, the eTextile output will not just depend on the sitting posture. In fact, the sensor value can be interfered by other uncertain factors, which make the textile sensor data difficult to be analyzed. Here, we list the dominant factors of signal distortion in eTextile. Basically, there are four different kinds of uncertain issues. Offset and scaling are caused by sheet-tosheet variation, and crosstalk and rotation are due to withinsheet variation.

1) Offset

In the ideal case, the initial pressure on each sensor should be zero. However, due to the sandwiched structure, initial offset pressure is unavoidable, and its value highly depends on the assembling method of the sensor. If the three layers are laminated tightly, the offset value will become high and vice versa. Obviously, different sheets should contain different assembling status, and this assembling variation implies different offset pressure values from sheet to sheet.

2) Scaling

The characteristics of eTextile have a large resistivity variation. Even if the same forces are applied to two similar eTextile sensors, their outputs are not necessarily the same. It is impossible to build up a general look-up table which describes the relationship between applied forces and sensor outputs in all cases. However, eTextile sensor is still promising for applications because the sensing repeatability and relativity are stable. It is possible to use a scientific method to predict the scaling variation.

3) Crosstalk

The crosstalk effect is one of the most challenging issues to deal with in textile sensors. Due to the limited space between sensors, all adjacent sensors are mechanically coupled together. As shown in Fig. 5, the initial sensor sheet is uniform, and the thickness value of each sensor is $H_{1}$. Once some force is applied on Sensor $S_{3}$, its thickness will be reduced to $\mathrm{H}_{2}$. In the meanwhile, the adjacent sensors also become squeezed more or less: the sensor values of $S_{2}$ and $S_{4}$ change because $S_{3}$ is pressed. The thickness of $S_{2}$ and $S_{4}$ changes to $H_{3}$, though there is no direct force applied on them. This phenomenon implies that the sensor readings are not only linked with the applied force but are also related to its neighboring sensors' conditions. 

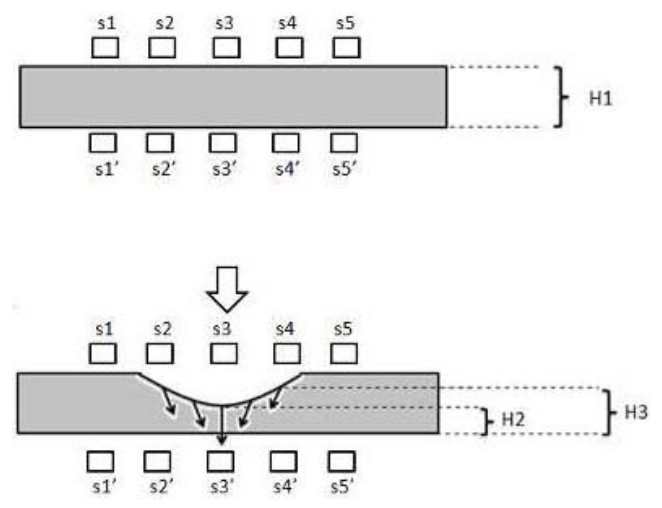

Fig. 5. An demostration of the crosstalk effect in the textile sensor array.

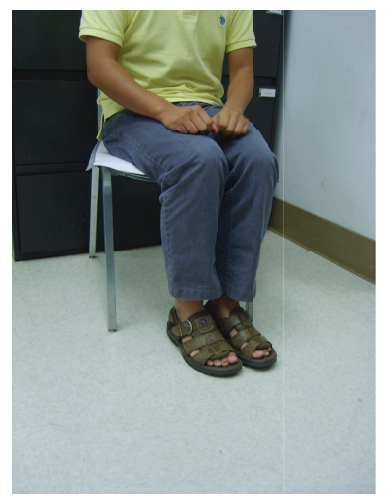

(a)

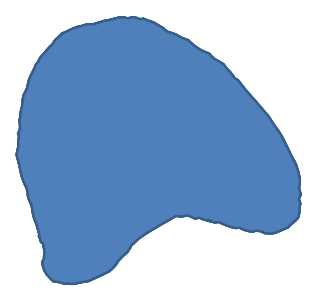

(c)

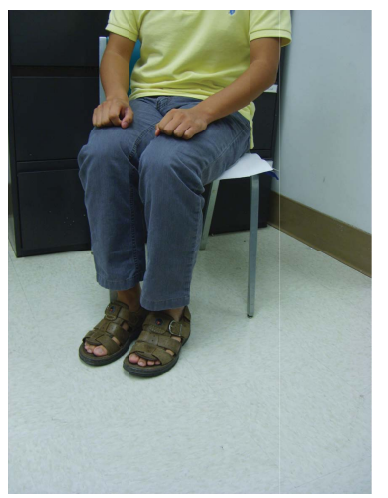

(b)

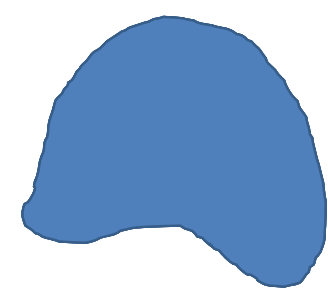

(d)
Fig. 6. The effect of the pressure map rotation. (a) Sitting $A$. (b) Sitting $B$. (c) Pressure map $A$. (d) Pressure map $B$.

\section{4) Rotation}

Even if the same user sits on Smart Cushion with the same gesture, the collected pressure map may not be the same due to the different sitting orientations. Fig. 6 shows two pressure distributions with the same gesture but different orientations. The pressure map in Fig. 6(c) is fairly similar to the one in Fig. 6(d). However, it is considerably difficult to match them with each other using pattern recognition techniques, since "rotation is always something hard to handle compared to translation and scaling" [28].

In this section, we presented the sensor design and imperfection of the textile sensor array. To achieve good performance in applications, all issues described above should be effectively handled. In the next section, we will introduce our proposed model to improve the quality of textile sensor data.

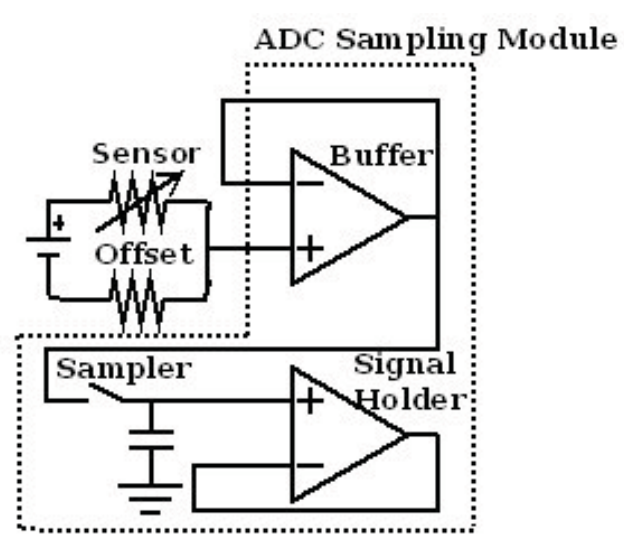

Fig. 7. Parametric modeling of a single textile sensor.

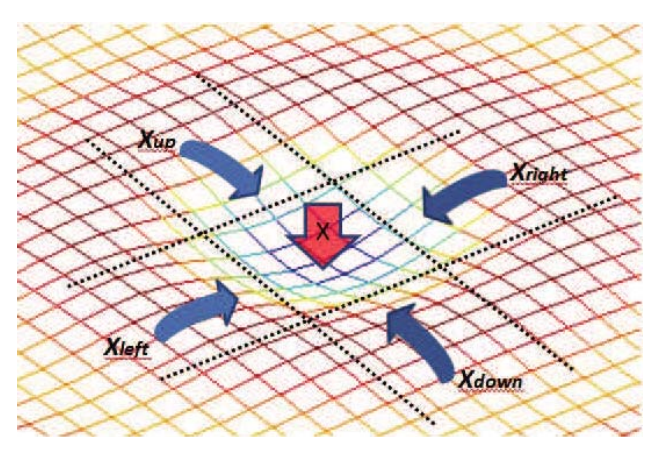

Fig. 8. The illustration of sensor $\mathrm{x}$ and its neighbors.

\section{SENSOR CALIBRATION}

\section{A. Preliminary}

To make reasonable inferences for applications, such as sitting position analysis, it is necessary to have accurate measurements from textile sensor. To address the uncertain issues we discussed from Section III-C, we analyzed the sensor signal model to learn the intrinsic relation between an applied force $F_{a}$ and a sensor reading $V_{o}$.

Fig. 7 models intrinsic parameters in a single textile sensor. The textile resistor, $R_{t}$, and the offset resistor, $R_{O}$ are connected in serial. One end is connected to a voltage supply, $V_{S}$, and the other is connected to the ground, GND. The middle joint point is connected to an ADC to obtain a sensor reading $V_{o}$. According to the mechanics of the eTextile [27], the extra applied force, $F_{a}$, is approximated to be inversely proportional to the sensor resistor $R_{t}$ :

$$
R_{t}=\frac{C}{F_{a}},
$$

where $C$ is a constant. Based on the parametric model in Fig. 7, we can derive a relational expression between sensor reading $S_{o}$ and applied force, $F_{a}$ :

$$
F_{a}=\frac{C \times V_{o}}{R_{t} \times\left(V_{s}-V_{o}\right)} .
$$

From Eq.2, we can see that the applied force is not linearly proportional to the ADC reading. This non-linearity makes the uncertain factors more difficult to deal with. 


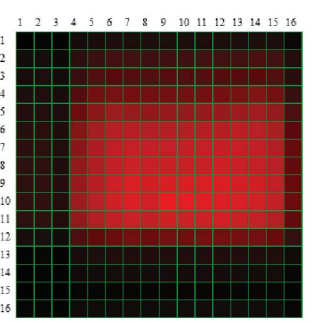

(a)

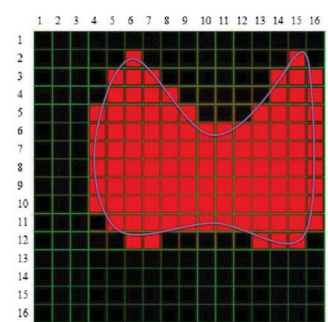

(b)

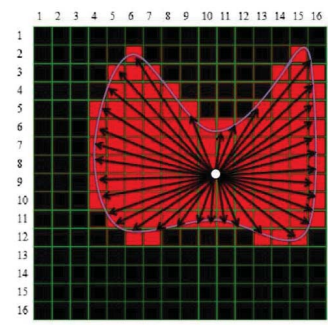

(c)

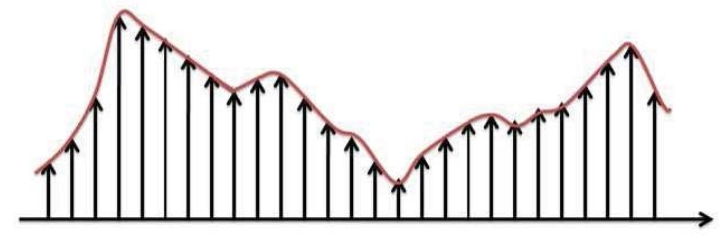

(d)

Fig. 9. Data representation procedure. (a) Map. (b) Boundary. (c) Distance. (d) Pressure profile sequence.

\section{B. MRF/Gibbs-Based Re-Sampling Method}

Our previous method to calibrate the sensors is to establish a pressure-resistance look-up table to exhaustively model the eTextile formulation via repeating voltage measurements under a number of imposed pressure values [29]. However, there are several difficulties involved. For example, even though we can measure the voltage of the voltage divider circuits one by one for each pressure sensor, we still cannot predict its output voltage if a number of its neighbor sensors are pressed at the same time because of the coupling effect mentioned in Section III-C. Furthermore, pressure-resistance relationship changes from one eTextile to another. Therefore, measuring each pressure-resistance relationship for each eTextile-based pressure sensor might result in an inefficient model.

In this paper, we proposed a new re-sampling based statistics technique under MRF/Gibbs distribution [30] to calibrate the uncertainties in eCushion, instead of directly building an ad-hoc modeling for each eTextile sensor. The basic idea is to find an up-threshold, $T h_{u p}$, a down-threshold, $T h_{d n}$, and the sensors that need to be calibrated. Firstly, we should decide which sensor needs to be re-sampled based on the following algorithm:

Here, we assume that the noise in Smart Cushion without any subject follows a Gaussian distribution. Once we have the re-sampling table Mask, we filter out the pressure values within the threshold range by randomly initializing the values. Then we start re-sampling under MRF/Gibbs probability

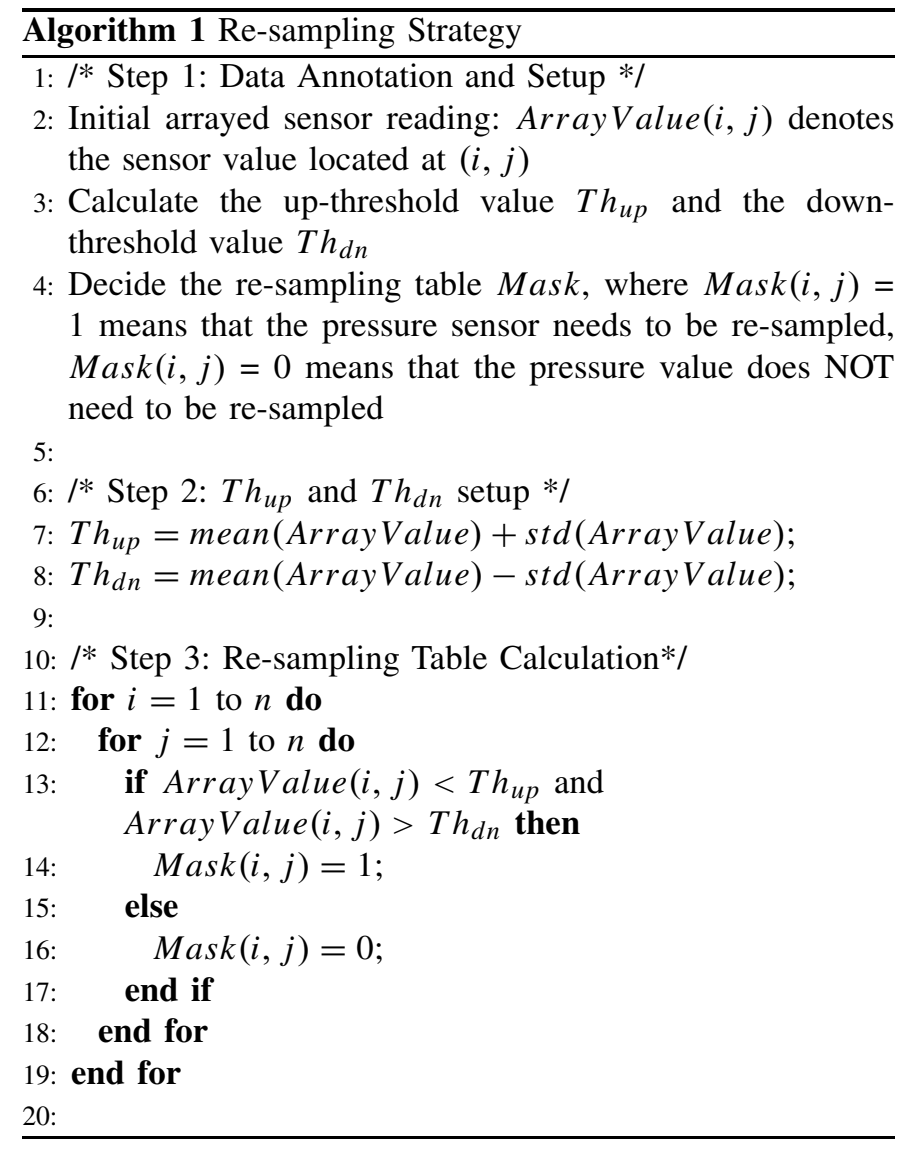

distribution as follows:

$$
P(X=x)=\frac{1}{Z(\beta)} e^{-\beta E(x)}
$$

In Eq. 3, $Z$ is used for normalizing the probability distribution. $E$ is the energy function derived from the neighboring pressure values around $x . \beta$ is a free variable for a finer tuning, such as using simulated annealing method. In our case we just decrease the $\beta$ value monotonously by a constant factor. Norm2 energy function is the summation of the squared differences between $x$ and its neighbors. The neighborhood definition is shown in Fig. 8. The energy function is denoted in Eq. 4 to calculate the probability density function of $x$ :

$$
p d f(x)=e^{-\beta\left(\left(x_{u p}-x\right)^{2}+\left(x_{d n}-x\right)^{2}+\left(x_{\text {left }}-x\right)^{2}+\left(x_{\text {right }}-x\right)^{2}\right)} .
$$

In our algorithm, whenever a corrupted pressure value detected out of $T h_{d n}$ and $T h_{u p}$, the probability distribution of the corrupted pressure value will be re-computed and the new value will be generated by the re-sampling formulation Eq. 3. Traveling all corrupted pressure values once is called 1-sweep. After a few sweeps, the corrupted pressure value will be closer to its neighboring pressure value, since the probability distribution will become narrower when the energy deceases. In other words, the newer sampling values drawn from the new probability distributions will be similar to their neighborhood pressure values.

Based on the above analysis, a heavier pressure point on the Smart Cushion system will have more impact on its 


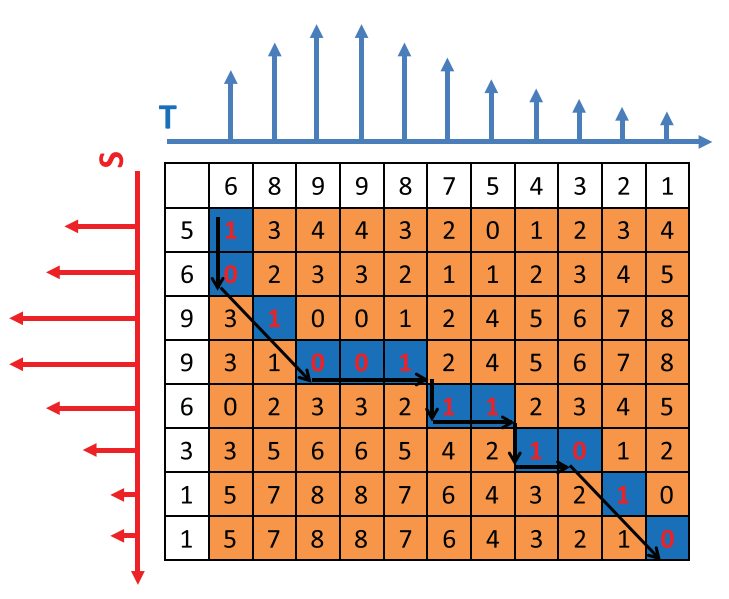

Fig. 10. A DTW example of two pressure profile sequences.

surrounding neighbors. It means that the pressure values inside the subject area will be boosted to a higher value close to the average pressure value of the heavy object imposed. This conclusion can be derived by the following proof:

Given that the boundary pressure value will be determined by the equation:

$$
p d f(x)=e^{-\sum_{i}\left(x_{\text {neighbor }}-x\right)^{2}} .
$$

The maximum likelihood can be obtained by its differentiation equation:

$$
\begin{aligned}
\frac{\partial p d f}{x} & =0, \\
\sum\left(x_{\text {neighbor }_{i}}-x\right)^{2} & =0 .
\end{aligned}
$$

Eq. 7 indicates that $x$ is more likely to be a value around the average of its neighbors. After applying the proposed algorithm, the holes inside the uniform weighted subject are filled. Less noisy pressure values will affect the summation result. Hence, the pressure estimation is approximately linear along with the weight of the subject on Smart Cushion. Since the threshold range is adaptive and only those pressure values in the suspicious zone will be modified, the proposed algorithm will not over-smooth the pressure readings.

In this section, we describe a re-sampling mechanism to iteratively adjust up and bottom threshold range based on a re-constructing probability distribution. After calibration, these sensors have higher precision, and the shape of the subject is also preserved. It is worth noticing that our method can be adapted in a variety of eTextile based sensors without manually tuning.

\section{Sitting Position Recognition}

A template-based algorithm [31] is proposed to distinguish sitting postures; however, it cannot handle the scenario where the sensing data is imperfect. In addition, Naive Bayes Network [32] method [15] is used to train the data and selected significantly featured sensors for classification. This method highly depends on the training data and cannot deal with the uncertain factors such as different weights, sizes and sitting orientations. In this section, we introduce our algorithm to efficiently address sitting posture analysis.

\section{A. Data Representation}

To facilitate the effort of pattern recognition, it is important to choose an appropriate way of data representation. Instead of dealing with pressure map (2D image) directly, we convert it into a pressure profile sequence (1D time series). In general, there are multiple advantages to work with $1 \mathrm{D}$ sequential data. First, the dimension of is data reduced; therefore, the algorithm complexity decreases dramatically (compared to 2D pressure image). Second, there are a number of existing algorithms to process $1 \mathrm{D}$ data sequences. Thirdly, it is much easier to tackle the rotation issue.

Our proposed procedures for data representation contain the following steps:

1) Step 1: Get the calibrated data as $2 D$ image shown in Fig.9(a).

2) Step 2: The Canny edge detector is applied to the data to obtain a binary image as shown in Fig. 9(b).

3) Step 3: Extracting the outline curve of the binary image. Note that it is always a closed curve due to the shape of human body.

4) Step 4: Measure the distance between every point and an image center (Fig. 9(c)).

5) Step 5: Signals can be treated as the Y-axis of a sequentially expanded signal waveform (Fig. 9(c)).

Fig. 9(a) shows the different steps of this method. After the last step, the pressure distribution information has been transformed to a 1D sequential data. In the next subsection, we will introduce a sitting posture recognition algorithm for this new data representation.

\section{B. Signal Matching Using Dynamic Time Warping}

We use dynamic time warping to classify different sitting postures. The targeted sequential signals are extracted from the corresponding pressure map while a user sits on Smart Cushion. Dynamic time warping $(D T W)$ is a similarity evaluation method of two time series signals. Compared to Euclidean distance, $D T W$ is more robust, allowing similar shapes to be matched even if they are out of phase [33].

Assuming there are two pressure sequences denoted as:

$$
\begin{gathered}
S=\left[s_{1}, s_{2}, s_{3}, \cdot, s_{i}, \cdot, s_{n}\right], \\
T=\left[t_{1}, t_{2}, t_{3}, \cdot, t_{j}, \cdot, t_{m}\right] .
\end{gathered}
$$

To evaluate the similarity of these two sequences, $D T W$ constructs a $n$ by $m$ matric $D$, where $d_{i j}=\left(s_{i}-t_{j}\right)^{2}$. Each element $d_{i j}$ denotes the similarity between $s_{i}$ and $t_{j}$. The main idea of $D T W$ is to find a continuous and monotonic path $W$ from $d_{11}$ to $d_{m n}$ with minimal cost. The time and space complexity of $D T W(S, T)$ is $\mathrm{O}(m n)$. Using LB_Keogh bounding can effectively make DTW run in $\mathrm{O}(\mathrm{n})$ [34], [35]. Fig. 10 shows an example of $D T W$-based similarity evaluation between two pressure profile sequences.

In order to speed up the algorithm, we set two bounds to reduce $D T W$ searching space. In this application, our 
TABLE I

The ExPerimental Results: Precision V.S. ReCALL

\begin{tabular}{|c|c|c|c|c|c|c|c|c|c|}
\hline & Situp & Forward & Backward & LL & RL & LFOR & RFOL & Total & Recall \\
\hline Situp & 85 & 7 & 8 & 0 & 0 & 0 & 0 & 100 & $85 \%$ \\
\hline Forward & 3 & 92 & 5 & 0 & 0 & 0 & 0 & 100 & $92 \%$ \\
\hline Backward & 9 & 4 & 87 & 0 & 0 & 0 & 0 & 100 & $87 \%$ \\
\hline LL & 1 & 2 & 0 & 74 & 0 & 15 & 8 & 100 & $74 \%$ \\
\hline RL & 1 & 1 & 4 & 0 & 82 & 1 & 11 & 100 & $82 \%$ \\
\hline LFOR & 0 & 0 & 0 & 5 & 1 & 90 & 4 & 100 & $90 \%$ \\
\hline RFOL & 1 & 1 & 1 & 2 & 3 & 2 & 91 & 100 & $91 \%$ \\
\hline Total & 100 & 107 & 105 & 81 & 86 & 108 & 114 & & \\
\hline Precision & $85 \%$ & $86 \%$ & $83 \%$ & $91 \%$ & $95 \%$ & $83 \%$ & $80 \%$ & & \\
\hline
\end{tabular}
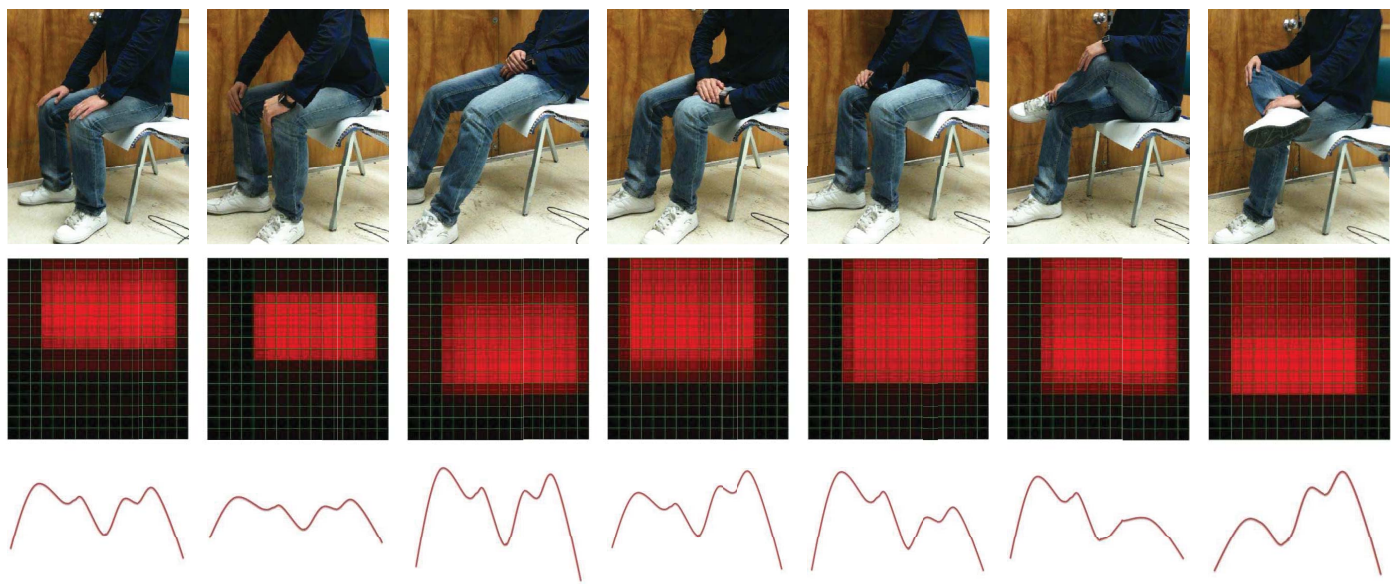

Fig. 11. Sitting posture analysis: seven sitting postures (top) are evaluated and each eTextile pressure map (middle) is transformed to a corresponding pressure profile sequence (bottom).

algorithm has adaptive bounding values according to the signal. Given any $2 r$ length subsequence $S_{\prime}^{\prime}=\left[s_{i-r}: s_{i+r}\right]$ in $S$, we can find that the upper bound of $S_{\prime}$ is $U_{i}$, and the lower bound of $S$ ' is $L_{i}$. The values of $U_{i}$ and $L_{i}$ are calculated as follows:

$$
\begin{aligned}
& U_{i}=1.5 \times \max \left(s_{i-r}: s_{i+r}\right), \\
& L_{i}=0.75 \times \min \left(s_{i-r}: s_{i+r}\right) .
\end{aligned}
$$

With these bound settings, $L B \_K \operatorname{eogh}(S, T)$ becomes:

$$
L B \_K \operatorname{Keogh}(S, T)=\sqrt{\sum\left\{\begin{array}{ccc}
\left(t_{i}-U_{i}\right)^{2} & \text { if } & t_{i}>U_{i} \\
\left(t_{i}-L_{i}\right)^{2} & \text { if } & t_{i}<L_{i} \\
0 & \text { otherwise }
\end{array}\right. \text { (12) }}
$$

Notice that, similar to [34], DTW similarity measurement will converge to the Euclidean distance when $r$ is 1 .

\section{EXPERIMENTS}

\section{A. Experimental Setup}

To evaluate the effectiveness of our Smart Cushion system, we performed a pilot study on-campus. There are 25 subjects participated in the experiments, including 15 males and 10 females. Each participant sits on Smart Cushion with seven most common sitting postures. These data are used as training data. The seven postures, considered for classification, include 1) situp, 2) forward, 3) backward, 4) left lean (LL), 5) right lean (RL), 6) right foot over left (RFOL) and 7) left foot over right (LFOR).
Fig. 11 shows an example indicating the procedure of data acquisition and preprocessing. The figures on the top row illustrate seven sitting postures. The middle row pictures show the corresponding pressure map of each posture. Each pressure map has 256 pixels in total. The bottom row pictures are the corresponding sequential representation using pressure maps. Each sequence is evaluated and classified using our proposed algorithm.

\section{B. Recognition Results}

The experimental results are shown in Table I. We list the recognition results of all sitting postures with the precision and recall calculation. The overall accuracy of our proposed algorithm over all sitting postures is $85.9 \%$, which outperforms the previous method (79\% in [29]). This is because the resampling based calibration method deals with the uncertainties in eTextile sensor data in a more accurate way. Furthermore, we notice that "situp", "forward" and "backward" postures are similar in the sense that their samples are never misclassified into other postures; however, they are confused with each other to a great extent. This observation indicates that these three sitting postures share many common features. This experience is helpful for us to further improve the algorithm performance while considering these sitting postures in particular.

\section{CONCLUSION}

In this paper, we design and implement the Smart Cushion system for sitting posture monitoring. In order to overcome 
several uncertain factors in eTextile sensors, including offset, scaling, crosstalk, and rotation effects, we propose a resampling based method to calibrate the sensor values. We also develop a dynamic time warping based algorithm to recognize different sitting postures. The experimental results show that the overall accuracy of Smart Cushion over all sitting positions is $85.9 \%$, which outperforms the previous method $(79 \%$ in [29]). We also learn that a number of sitting postures are similar to each other and harder to be differentiated from each other. In the future, we will attempt to improve the recognition rate with the experience we learned. Meanwhile, we consider to further scale up our system for other applications such as a bedsheet for sleeping monitoring and a carpet for gait analysis.

\section{REFERENCES}

[1] S. Haynes and K. Williams, "Impact of seating posture on user comfort and typing performance for people with chronic low back pain," Int. J. Ind. Ergonom., vol. 4, pp. 186-194, Jan. 2008.

[2] H. K. A. Maria Lis, K. M. Black, and M. Nordin, "Association between sitting and occupational LBP," Eur. Spine J., vol. 16, pp. 283-298, Nov. 2007.

[3] B. Magnas, "Body position and cerebrospinal fluid pressure," J. Neurosurgery, vol. 44, pp. 687-697, Jun. 1976.

[4] S. Perlmutter, F. Lin, and M. Makhsous, "Quantitative analysis of static sitting posture in chronic stroke," Gait Model., vol. 10, pp. 13-17, Jun. 2010.

[5] J. Deutsch, M. Borbely, J. Filler, K. Huhn, and P. Guarrera-Bowlby, "Use of a low-cost, commercially available gaming console (Wii) for rehabilitation of an adolescent with cerebral palsy," Phys. Therapy, vol. 88, pp. 1196-1207, Oct. 2008.

[6] M. Marschall, A. Harrington, and J. Steele, "Effect of work station design on sitting posture in young children," Ergonomics, vol. 4, pp. 1932-1940, Sep. 1995.

[7] K. Kamiya, M. Kudo, H. Nonaka, and J. Toyama, "Sitting posture analysis by pressure sensors," in Proc. Int. Conf. Pattern Recognit., Dec. 2009, pp. 155-162.

[8] J. Meyer, P. Lukowicz, and G. Troster, "Textile pressure sensor for muscle activity and motion dection," in Proc. Int. Conf. Wearable Comput., Sep. 2007, pp. 34-41.

[9] J. Liu, W. Xu, M.-C. Huang, N. Alshurafa, and M. Sarrafzadeh, "A dense pressure sensitive bedsheet desgin for unobtrusive sleep posture monitoring," in Proc. IEEE Int. Conf. Pervas. Comput. Commun., Mar. 2013, pp. 1-9.

[10] W. Xu, M.-C. Huang, N. Amini, J. Liu, L. He, and M. Sarrafzadeh, "Smart Insole: A wearable system for gait analysis," in Proc. Int. Conf. Pervas. Technol. Rel. Assist. Environ., Jun. 2012, pp. 1-4.

[11] J. Farringdona, A. J. Moorea, N. Tilburyb, J. Churchb, and P. D. Biemond, "Wearable sensor badge and sensor jacket for context awareness," in Proc. Int. Symp. Wearable Comput., Sep. 1999, pp. $107-113$.

[12] M.-C. Huang, W. Xu, Y. Su, C.-Y. Chang, B. Lange, and M. Sarrafzadeh, "Smart glove for upper extremities rehabilitative gaming assessment," in Proc. Int. Conf. Pervas. Technol. Rel. Assist. Environ., Jun. 2012, pp. $1-4$.

[13] M.-C. Huang, E. Chen, W. Xu, M. Sarrafzadeh, B. Lange, and C.-Y. Chang, "Gaming for upper extremities rehabilitation," in Proc. ACM/EMS Conf. Wireless Health, Oct. 2011, pp. 1-2.

[14] C. Mattmann, F. Clemens, and G. Troster, "Sensor for measuring strain in textile," Sensors, vol. 8, pp. 3719-3732, Jun. 2008.

[15] J. Meyer, B. Arnrich, J. Schumm, and G. Troster, "Design and modeling of a textile pressure sensor for sitting posture analysis," IEEE Sensor J., vol. 10, no. 8, pp. 1391-1398, Aug. 2010.

[16] C. R. Merritt, H. T. Nagle, and E. Grant, "Textile-based capacitive sensors for respiration monitoring," IEEE Sensors J., vol. 9, no. 1, pp. 71-78, Jan. 2009.

[17] J. Witt, F. Narbonneau, M. Schukar, K. Krebber, J. D. Jonckeere, M. Jeanne, D. Kinet, B. Paquet, A. Depre, L. T. D'Angelo, T. Thiel, and R. Logier, "Medical textiles with embedded fiber optic sensors for monitoring of respiratory movement," IEEE Sensors J., vol. 12, no. 1, pp. 246-254, Jan. 2012.
[18] M.-C. Huang, W. Xu, J. Liu, L. Samy, and M. Sarrafzadeh, "Inconspicuous on-bed respiration monitoring," in Proc. Int. Conf. Pervas. Technol. Rel. Assist. Environ., 2013, pp. 1-8.

[19] S. Kim, S. Leonhardt, N. Zimmermann, P. Kranen, D. Kensche, E. Muller, and C. Quix, "Influence of contact pressure and moisture on the signal quality of a newly developed textile ECG sensor shirt," in Proc. Int. Conf. Wearable Implant. Body Sensor Netw., May 2008, pp. 28-33.

[20] Y. Zhang, C. C. Poon, C. H. Chan, M. W. Tsang, and K.-F. Wu, "A health-shirt using e-textile materials for the continuous and cuffless monitoring of arterial blood pressure," in Proc. Int. Summer School Symp. Med. Devices Biosensors, Sep. 2006, pp. 18-23.

[21] J. Liu, M.-C. Huang, W. Xu, N. Alshursfa, and M. Sarrafzadeh, "On-bed monitoring for range of motion exercises with a pressure sensitive bedsheet," in Proc. IEEE Conf. Body Sensor Netw., May 2013, pp. $325-330$.

[22] Tekscan. (2013, Jun.), Alpharetta, GA, USA [Online]. Available: http://www.tekscan.com/

[23] M.-C. Huang, W. Xu, J. Liu, L. He, Y. Su, and M. Sarrafzadeh, "Inconspicuous personal computer protection with touch-mouse," in Proc. Int. Conf. Human Comput. Interact., 2013, pp. 20-29.

[24] C. Mattmann, O. Amft, H. Harms, G. Troster, and F. Clemens, "Recognizing upper body postures using textile strain sensors," in Proc. IEEE Int. Symp. Wearable Comput., Oct. 2007, pp. 463-466.

[25] E. Mitchell, S. Coyle, N. Connor, and D. Diamond, "Breathing feedback system with wearable textile sensors," in Proc. IEEE Conf. Body Sensor Netw., Jun. 2010, pp. 56-61.

[26] L. Shu, T. Hua, Y. Wang, Q. Li, F. Dong, and X. Tao, "In-shoe plantar pressure measurement and analysis system based on fabric pressure sensing array," IEEE Trans. Inf. Technol. Biomed., vol. 14, no. 3, pp. 767-775, May 2010.

[27] M. Rofouei, W. Xu, and M. Sarrafzadeh, "Computing with uncertainty in a smart textile surface for object recognition," in Proc. IEEE Int. Conf. Multisensor Fusion Integr. Intell. Syst., Sep. 2010, pp. 50-65.

[28] D. Li and S. Simske, "Shape retrieval based on distance ratio distribution," HP Laboratories, Palo Alto, CA, USA, Tech. Rep. HPL-2002-251, Sep. 2002.

[29] W. Xu, Z. Li, M. Huang, N. Amini, and M. Sarrafzadeh, "eCushion: An eTextile device for sitting posture monitoring," in Proc. Int. Conf. Body Sensor Netw., May 2011, pp. 194-199.

[30] R. M. Mohamed, A. El-Baz, and A. A. Farag, "Image modeling using gibbs-Markov random field and support vector machines algorithm," Int. J. Inf. Technol., vol. 1, no. 4, pp. 1932-1940, Nov. 2004.

[31] M. Zhu, A. Martinez, and H. Tan, "Template-based recognition of static sitting postures," in Proc. Comput. Vis. Pattern Recognit. Workshop, Jun. 2003, pp. 50-53.

[32] A. McCallum and K. Nigam, "A comparison of event models for naive bayes text classification," in Proc. Int. Conf. AAAI, Sep. 1998, pp. 107-113.

[33] E. Keogh and C. Ratanamahatana, "Exact indexing of dynamic time warping," in Proc. ACM Int. Conf. Very Large Data Bases, Sep. 2002, pp. 92-111.

[34] E. Keogh, L. Wei, X. Xi, S. H. Lee, and M. Vlachos, "LB Keogh supports exact indexing of shapes under rotation invariance with arbitrary representations and distance measures," in Proc. ACM Int. Conf. Very Large Data Bases, Sep. 2006, pp. 56-78.

[35] A. A. Smith and M. Craven, "Fast multisegment alignments for temporal expression profiles," in Proc. 7th Int. Conf. Comput. Syst. Bioinf., 2008, pp. $315-326$.

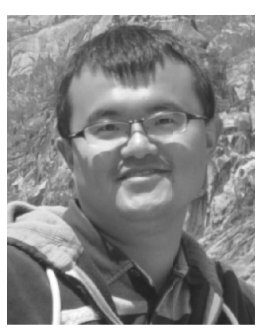

Wenyao Xu received the Ph.D. degree from the Electrical Engineering Department, University of California, Los Angeles, CA, USA, in 2013. Currently, he is an Assistant Professor with the Computer Science and Engineering Department, University at Buffalo, Buffalo, NY, USA, State University of New York, New York, NY, USA. His current research interests include embedded system design, computational modeling, algorithm design, humancomputer interaction, integrated circuit design technologies, and their applications in medical and health

applications

He received the Best Medical \& Performance Application Paper Award of the IEEE Conference on Implantable and Wearable Body Sensor Networks in 2013 and the Best Demonstration Award of ACM Wireless Health Conference in 2011 . 


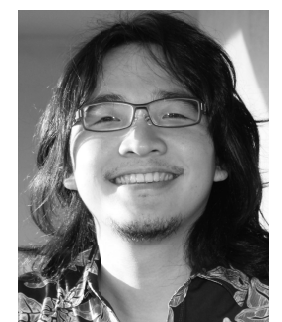

Ming-Chun Huang is currently pursuing the Ph.D. degree with the Computer Science Department, University of California, Los Angeles (UCLA), CA, USA, and the B.S. degree in electrical engineering from National Tsing Hua University, Hsinchu, Taiwan, and the M.S. degree in electrical engineering from the University of Southern California, Los Angeles. His current research interests include human-computer interacting behavior modeling, inconspicuous sensing, named data networking, and their applications of smart home and nursing

home environment healthcare.

He received the Best Medical \& Performance Application Paper Award of the IEEE Conference on Implantable and Wearable Body Sensor Networks in 2013 and the Best Demonstration Award in ACM Wireless Health Conference in 2011. He was granted for his long-term contribution of undergraduate education at UCLA in 2013. He has five patents resulting from his research work were licensed to a startup company of healthcare technology: Medisens, Santa Clara, CA. He has served as a Co-Founder and a Senior Engineering Consultant with Medisens.

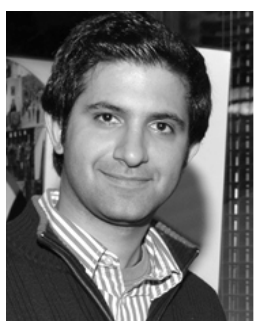

Navid Amini is a Post-Doctoral Fellow with the Jules Stein Eye Institute, University of California, Los Angeles (UCLA), CA, USA, where he investigates different sensing technologies and signal processing techniques to quantify the quality of life for people with low vision. He received the B.Sc. degree in computer engineering from Sharif University, Tehran, Iran, in 2007, and the M.Sc. and $\mathrm{Ph} . \mathrm{D}$. degrees in computer science from UCLA in 2010 and 2012, respectively. His current research on biomedical signal processing.

interests include mobile computing with emphasis

He is a recipient of the CTSI Core Voucher Award at UCLA. He received the Symantec Outstanding Graduate Student Research Award and the Google Outstanding Graduate Student Research Award. He currently serves as a Technical Program Committee Member for the 8th International Conference on Body Area Networks.

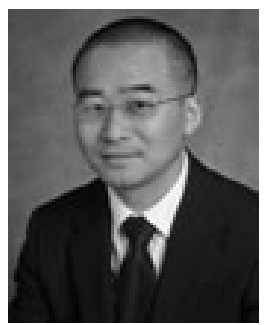

Lei He is a Professor of Electrical Engineering Department, University of California, Los Angeles (UCLA), CA, USA, and was a Faculty Member with the University of Wisconsin, Madison, WI USA, in 1999 and 2002. He was a Visiting or Consulting positions with Cadence, San Jose, CA, USA, Empyrean Soft, Hewlett-Package, Palo Alto, CA, Intel, and Synopsys, San Jose, and a Technical Advisory Board Member for Apache Design Solutions and Rio Design Automation.

$\mathrm{He}$ received the $\mathrm{Ph} . \mathrm{D}$. degree in computer science from UCLA in 1999. His current research interests include modeling and simulation, VLSI circuits and systems, and cyber physical systems. He has published one book and over 200 technical papers with 12 best paper nominations mainly from Design Automation Conference and International Conference on Computer-Aided Design and five Best Paper or Best Contribution Awards, including the ACM Transactions on Electronic System Design Automation 2010 Best Paper Award.

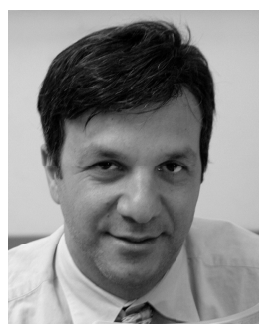

Majid Sarrafzadeh received the Ph.D. degree in electrical and computer engineering from the University of Illinois at Urbana-Champaign, Urbana, IL, USA, in 1987. He joined Northwestern University, Evanston, IL, USA, as an Assistant Professor, in 1987. In 2000, he joined the Computer Science Department, University of California at Los Angeles (UCLA), Los Angeles, CA, USA. He is a CoFounder and a Co-Director of the UCLA Wireless Health Institute. His current research interests include embedded computing with emphasis on healthcare. He has published over 400 papers, co-authored five books, and is a named inventor on many U.S. patents. He has collaborated with many industries in the past 25 years. He co-founded two companies over 2000 they were both acquired around 2004. He has recently co-founded MediSens and Bruin Biometrics, both in the area of Technology in Healthcare. 